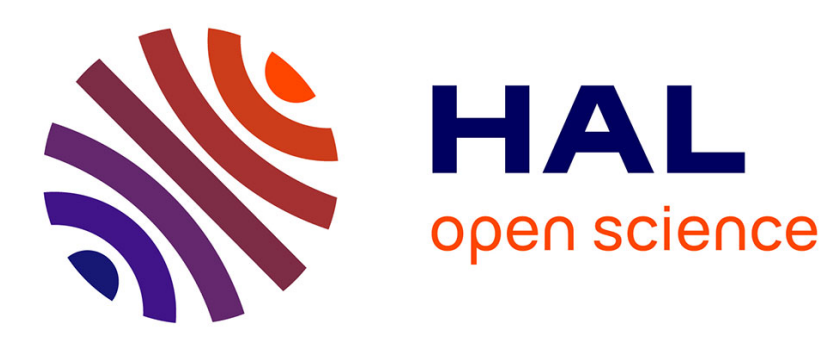

\title{
Size-Effects at a Crack-Tip Interacting with a Number of Voids
}

Viggo Tvergaard, Christian F. Niordson

\section{To cite this version:}

Viggo Tvergaard, Christian F. Niordson. Size-Effects at a Crack-Tip Interacting with a Number of Voids. Philosophical Magazine, 2009, 88 (30-32), pp.3827-3840. 10.1080/14786430802225540 . hal00513908

\section{HAL Id: hal-00513908 \\ https://hal.science/hal-00513908}

Submitted on 1 Sep 2010

HAL is a multi-disciplinary open access archive for the deposit and dissemination of scientific research documents, whether they are published or not. The documents may come from teaching and research institutions in France or abroad, or from public or private research centers.
L'archive ouverte pluridisciplinaire HAL, est destinée au dépôt et à la diffusion de documents scientifiques de niveau recherche, publiés ou non, émanant des établissements d'enseignement et de recherche français ou étrangers, des laboratoires publics ou privés. 


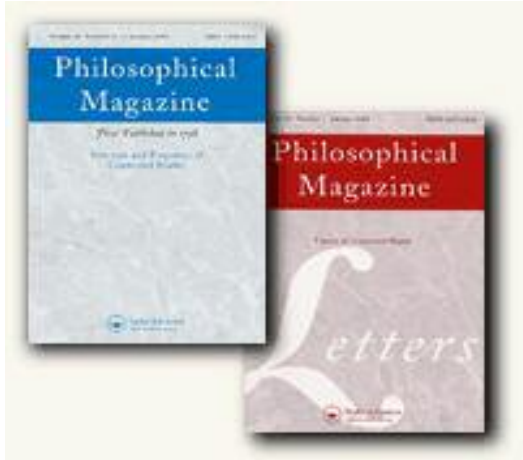

\section{Size-Effects at a Crack-Tip Interacting with a Number of Voids}

\begin{tabular}{|c|c|}
\hline Journal: & Philosophical Magazine \& Philosophical Magazine Letters \\
\hline Manuscript ID: & TPHM-07-Nov-0337.R1 \\
\hline Journal Selection: & Philosophical Magazine \\
\hline $\begin{array}{r}\text { Date Submitted by the } \\
\text { Author: }\end{array}$ & 08-Apr-2008 \\
\hline Complete List of Authors: & $\begin{array}{l}\text { Tvergaard, Viggo; Technical University of Denmark } \\
\text { Niordson, Christian; Technical University of Denmark, Department } \\
\text { of Mechanical Engineering, Solid Mechanics }\end{array}$ \\
\hline Keywords: & crack growth, plasticity \\
\hline Keywords (user supplied): & ductile fracture, finite strains, voids \\
\hline
\end{tabular}

\section{\$) ScholarONE" \\ Manuscript Central}




\title{
Size-Effects at a Crack-Tip Interacting with a Number of Voids
}

\author{
VIGGO TVERGAARD AND CHRISTIAN F. NIORDSON
}

Department of Mechanical Engineering, Solid Mechanics

Technical University of Denmark, DK-2800 Kgs. Lyngby, Denmark

\begin{abstract}
A strain gradient plasticity theory is used to analyze the growth of discretely represented voids in front of a blunting crack-tip, in order to study the influence of size-effects on two competing mechanisms of crack growth. For a very small void volume fraction the crack-tip tends to interact with one void at a time, while larger void volume fractions lead to simultaneous interaction of multiple voids on the plane ahead of the crack-tip. The present computations are stopped before coalescence of the nearest void with the crack-tip. Analyses are carried out for different values of the characteristic material length relative to the initial void radius. For a case showing the multiple void mechanism it is found that the effect of the material length can change the behaviour towards the void by void mechanism. A material model with three characteristic length scales is compared with a one length scale model and the differences are discussed.
\end{abstract}

Keywords: Plasticity; finite strains; ductile fracture; crack growth; voids.

\section{Introduction}

The mechanism of crack growth in a ductile material is usually void growth to coalescence ahead of the crack-tip. The initiation of this process has been studied by analyses of the interaction between a blunting crack-tip and a neighbouring void by Rice and Johnson [1], McMeeking [2], Aravas and McMeeking [3] and Hom and McMeeking [4]. Using constitutive models for a porous ductile material, such as that of Gurson [5], is an alternative approach for studying the effect of voids near a crack-tip as analyzed by Needleman and Tvergaard, [6], Rousselier, [7], Brocks et al. [8], Gao et al. [9], Tvergaard and Needleman, [10].

Tvergaard and Hutchinson [11] have considered a multiple void version of the earlier plane strain studies to analyze ductile crack growth by discrete representation of up to six voids ahead of the tip. When there is only a single void ahead of the crack-tip, the focus is on the growth of this void in the large strain field that sweeps over the material ahead of the blunting crack-tip, and finally on the coalescence of the void with the crack front. However, the analysis with six voids ahead of the tip illustrated a competition between two mechanisms of ductile crack growth. For sufficiently low void volume fractions the blunting crack-tip first interacts with the nearest void until they have coalesced, then with the next void, etc., which defines the void 
by void mechanism. The second mechanism involves the simultaneous interaction of multiple voids on the plane ahead of the crack-tip, both during initiation and during the subsequent crack growth. This multiple void mechanism was found to be active for somewhat larger void volume fractions. Recently, these mechanisms have been further studied (Tvergaard [12]) by considering a larger number of discretely represented voids and by using remeshing to follow the ligament necking process in more detail. Discretely represented cylindrical voids ahead of the crack-tip have also been considered by Petti and Dodds [13] with focus on the effect of the voids and the crack growth mechanism on cleavage fracture, using the Weibull stress model to quantify the effect of stress concentrations in the void growth region on the probability of cleavage fracture.

In the present paper the effect of strain gradient plasticity is incorporated in analyses of several discretely represented voids interacting with a blunting crack-tip. Studies of the growth of very small voids have shown that the rate of growth is much reduced when the void radius is smaller than the characteristic material length incorporated in the nonlocal plasticity theory (Fleck and Hutchinson [14], Huang et al. [15], Niordson and Tvergaard $[16,17])$. Here, the analyses consider the possibility that the competition between the void by void mechanism of crack growth and the multiple void mechanism of crack growth is affected by the size effects, since the growth of individual voids is affected. In addition to the material lengths incorporated in the nonlocal material model the problem to be analyzed here also includes several other length scales, i.e. the initial spacing of the discretely represented voids, the initial radius of these voids, and the initial crack-tip radius, and the results involve the interaction of all these characteristic lengths.

\section{Problem Formulation}

A finite strain generalization [18] for the strain gradient plasticity theory by Fleck and Hutchinson [14] is used to model the material numerically using an updated Lagrangian formulation, with a Cartesian frame as reference.

The theory incorporates gradient hardening through invariants of the gradient of the plastic strain rate $\rho_{i j k}=\rho_{j i k}=\dot{\varepsilon}_{i j, k}^{P}$. The basic idea in the theory is that plastic work is expended through a gradient enhanced measure of effective plastic strain, $E^{P}$, defined incrementally through

$$
\dot{E}^{P^{2}}=\dot{\varepsilon}^{P^{2}}+l_{1}^{2} I_{1}+4 l_{2}^{2} I_{2}+\frac{8}{3} l_{3}^{2} I_{3}
$$

Here, $\dot{\varepsilon}^{P^{2}}=\frac{2}{3} \dot{\varepsilon}_{i j}^{P} \dot{\varepsilon}_{i j}^{P}$ is the conventional measure of effective plastic strain, $I_{1}, I_{2}$ and $I_{3}$ are the three nonzero invariants of homogeneous degree two of $\rho_{i j k}$. For dimensional consistency three length parameters $l_{1}, l_{2}$ and $l_{3}$ are introduced. Decomposing the plastic strain rate, $\dot{\varepsilon}_{i j}^{P}=m_{i j} \dot{\varepsilon}^{P}$, into its direction, $m_{i j}$, and its magnitude $\dot{\varepsilon}^{P}$, yields the following alternative form of Eq. (1)

$$
\dot{E}^{P^{2}}=\dot{\varepsilon}^{P^{2}}+A_{i j} \dot{\varepsilon}_{, i}^{P} \dot{\varepsilon}_{, j}^{P}+B_{i} \dot{\varepsilon}_{, i}^{P} \dot{\varepsilon}^{P}+C \dot{\varepsilon}^{P^{2}}
$$

where the tensors $A_{i j}, B_{i}$ and $C$ depend on the plastic strain rate direction, $m_{i j}$, and its spatial gradient, $m_{i j, k}$. Detailed expressions for these tensors can be found in [14].

The theory is based on the second order plastic work per unit volume given by the expression 


$$
h\left[E^{P}\right] \dot{E}^{P^{2}}
$$

Hence, plastic work is expended through both plastic deformation as measured by $\dot{\varepsilon}^{P}$ as well as the gradient of the plastic strain rate $\rho_{i j k}=\dot{\varepsilon}_{i j, k}^{P}$ through the invariants in Eq. (1).

For a body of volume $V$ with the surface $S$, the weak form of the equilibrium equations for the strain gradient plasticity theory is given by the principle of virtual work in the current configuration

$$
\int_{V}\left(\sigma_{i j} \delta \dot{\varepsilon}_{i j}-\left(Q-\sigma_{(e)}\right) \delta \dot{\varepsilon}^{P}+\tau_{i} \delta \dot{\varepsilon}_{, i}^{P}\right) d V=\int_{S}\left(T_{i} \delta \dot{u}_{i}+t \delta \dot{\varepsilon}^{P}\right) d S
$$

Here, $\sigma_{i j}$ is the Cauchy stress tensor work conjugate to the total strain rate $\dot{\varepsilon}_{i j}=\frac{1}{2}\left(\dot{u}_{i, j}+\dot{u}_{j, i}\right)$, where $\dot{u}_{i}$ is the displacement rate, $Q$ is a generalized effective stress, $\sigma_{(e)}=\sqrt{\frac{3}{2} S_{i j} S_{i j}}$ is von Mises effective stress, and $S_{i j}=\sigma_{i j}-\delta_{i j} \frac{\sigma_{k k}}{3}$ is the stress deviator, with $\delta_{i j}$ denoting Kronecker's delta. The surface integral contains traction contributions from the conventional surface traction $T_{i}$ work conjugate to the displacement rate $\dot{u}_{i}$ and a higher order traction, $t$, work conjugate to the effective plastic strain rate, $\dot{\varepsilon}^{P}$.

The plastic strain increment is defined according to the usual relation for $J_{2}$ flow theory

$$
\dot{\varepsilon}_{i j}^{P}=\frac{3}{2} \frac{S_{i j}}{\sigma_{(e)}} \dot{\varepsilon}^{P}=m_{i j} \dot{\varepsilon}^{P}
$$

so that $m_{i j}$ is chosen as the direction of the stress deviator.

Introducing Kirchhoff stress measures the principle of virtual work can be expressed in the reference configuration in an updated Lagrangian framework by

$$
\int_{V}\left({ }^{\nabla} \varsigma_{i j} \delta \dot{\varepsilon}_{i j}-\sigma_{i j}\left(2 \dot{\varepsilon}_{i k} \delta \dot{\varepsilon}_{k j}-\dot{e}_{k j} \delta \dot{e}_{k i}\right)+\left(\dot{q}-\dot{\sigma}_{(e)}^{\varsigma}\right) \delta \dot{\varepsilon}^{P}+\stackrel{\vee}{\rho}_{i} \delta \dot{\varepsilon}_{, i}^{P}\right) d V=\int_{S}\left(\dot{T}_{0 i} \delta \dot{u}_{i}+\dot{t}_{0} \delta \dot{\varepsilon}^{P}\right) d S
$$

where $\varsigma_{i j}^{\nabla}$ is the Jaumann rate of the Kirchhoff stress, $\dot{q}$ is the rate of the Kirchhoff variant of the effective stress, which is work conjugate to $\varepsilon^{P}$, and $\stackrel{\vee}{\rho}_{i}$ is the convected derivative of the higher order Kirchhoff stress. The displacement gradient is denoted $\dot{e}_{i j}=\dot{u}_{i, j}$. The Kirchhoff stress is related to the Cauchy stress by $\varsigma_{i j}=J \sigma_{i j}$, the higher order Kirchhoff stress is related to the true higher order stress by $\rho_{i}=J \tau_{i}$, and $q=J Q$, where $J$ is the determinant of the metric tensor. The effective stress enters the principle of virtual work in the updated configuration through $\sigma_{(e)}^{\varsigma}=J \sigma_{(e)}$. 
In the right-hand side of Eq. (6) $\dot{T}_{0 i}$ and $\dot{t}_{0}$ are nominal traction rates conjugate to $u_{i}$ and $\varepsilon^{P}$, respectively. In addition to conventional boundary conditions, conditions on the higher order traction rate $\dot{t}_{0}$ or the effective plastic strain rate $\dot{\varepsilon}^{P}$ are needed. For the present problem $\dot{t}_{0}=0$ is imposed for all higher order boundary conditions, on internal boundaries as well as external boundaries. At the free surfaces this models free passage of dislocations through the boundary. The use of the natural boundary condition $\left(\dot{t}_{0}=0\right)$ at internal boundaries implies no constraint on plastic flow, which suggest that dislocations are free to pass through the current elastic-plastic boundary. The physical interpretation of higher order boundary conditions is presently the focus of much research. For further discussions on higher order boundary conditions see $[14,20,21,22]$.

The constitutive equations for the various Kirchhoff stress measures are

$$
\begin{gathered}
\varsigma_{i j}^{\nabla}=R_{i j k l}\left(\dot{\varepsilon}_{k l}-\dot{\varepsilon}^{P} m_{k l}\right)=\dot{\zeta}_{i j}-\dot{\omega}_{i k} \sigma_{k j}-\sigma_{i k} \dot{\omega}_{j k} \\
\dot{q}=h\left(\dot{\varepsilon}^{P}+\frac{1}{2} B_{i} \dot{\varepsilon}_{, i}^{P}+C \dot{\varepsilon}^{P}\right) \\
\check{\rho}_{i}=h\left(A_{i j} \dot{\varepsilon}_{, j}^{P}+\frac{1}{2} B_{i} \dot{\varepsilon}^{P}\right)=\dot{\rho}_{i}-\dot{e}_{i k} \rho_{k}
\end{gathered}
$$

where $h=h\left[E^{p}\right]$ is the hardening modulus, which is evaluated at $E^{p}$, rather than at $\varepsilon^{p}$ (as it would in conventional $J_{2}$ flow theory), $\dot{\omega}_{i j}$ is the antisymmetric part of $\dot{e}_{i j}$ and

$$
R_{i j k l}=\frac{E}{1+v}\left(\frac{1}{2}\left(\delta_{i k} \delta_{j l}+\delta_{i l} \delta_{j k}\right)+\frac{v}{1-2 v} \delta_{i j} \delta_{k l}\right)
$$

is the elastic stiffness tensor. Here, $E$ is Young's modulus and $v$ is Poisson's ratio.

The general theory proposed by Fleck and Hutchinson [14], employs the three constitutive length parameters $l_{1}, l_{2}$ and $l_{3}$ to scale the influence of the three invariants of homogeneous degree two of the gradient of the plastic strain rate $\rho_{i j k}=\dot{\varepsilon}_{i j, k}^{P}$, of which $l_{1}$ can be identified as dominating gradients of stretch, while $l_{2}$ and $l_{3}$ dominate when rotation gradients appear. A simplified model, which does not distinguish gradients of stretch and rotation can be encompassed within the general framework of the present model by introducing a new definition of the gradient enhanced measure of effective plastic strain according to the incremental relation

$$
\dot{E}^{P^{2}}=\dot{\varepsilon}^{P^{2}}+l_{*}^{2} \dot{\varepsilon}_{, i}^{P} \dot{\varepsilon}_{, i}^{P}
$$

Here, $l_{*}$ is a new length parameter. In this particular case the tensors in the constitutive relations for the generalized effective stress and the higher order have the simple expressions $A_{i j}=l_{*}^{2} \delta_{i j}, B_{i}=0$ and $C=0$. The theory embodied by this definition of the gradient enhanced measure of effective plastic strain is closely related to the original gradient model by Aifantis [19]. For further details on the strain gradient theory and the finite strain generalization see [14,18].

As in Tvergaard and Hutchinson [11] and Tvergaard [12] the plane strain analyses are carried out for pure mode I loading under conditions of small scale yielding. Thus, on the outer edge of the region analyzed 
numerically displacement increments are prescribed according to the elastic singular $K_{I}$ field. A Cartesian $x^{i}$ coordinate system is centered at the initial crack-tip, with the $x^{1}$ axis in the crack plane, in the direction of crack growth, and due to symmetry only the region for $x^{2}>0$ is analyzed numerically. The initial geometry of the crack-tip region (see Fig. 1) is specified by the radius $r_{0}$ of the crack-tip, the radius $R_{0}$ of the cylindrical voids, and the spacing $X_{0}$ between the centres of the voids and between the crack-tip and the first void. The initial void volume fraction $f_{0}$ is defined as that in a strip of material of width $X_{0}$, which gives $f_{0}=\pi\left(R_{0} / X_{0}\right)^{2}$. The present analyses are carried out with four discrete cylindrical voids represented ahead of the crack-tip.

The numerical solutions are obtained using a two-field finite element method similar to that used by de Borst and Mühlhaus [23]. In the present implementation following Niordson and Tvergaard [24] isoparametric eight-noded elements are used for the displacement fields as well as for the plastic strain field. The same elements have been used by Komaragiri et al. [25] in a study of crack-tip behaviour with strain gradient plasticity. An element with only four-noded elements for the plastic strain field, as suggested by Mikkelsen [26], has been tried for a couple of the cases analyzed, but was not found advantageous, since very small increments were needed.

When $K_{I}$ is the current value of the stress singularity, giving the current displacements specified as boundary conditions at the outer edge, the value of the $J$ integral is

$$
J=\frac{1-v^{2}}{E} K_{I}^{2}
$$

In the results presented here the current value of the load is specified in terms of the dimensionless parameter $J / \sigma_{Y} X_{0}$, where $\sigma_{Y}$ is the initial yield stress.

Along the crack plane symmetry boundary conditions are specified, while on the crack surface and on the void surfaces the values of the nominal tractions in the right-hand side of (5) are taken to be zero.

Strain hardening is modelled according to the following expression for the hardening modulus

$$
h\left[E^{P}\right]=\left(\frac{1}{E_{T}}-\frac{1}{E}\right)^{-1}
$$

where the tangent modulus is given by

$$
E_{T}=\frac{E}{n}\left(\frac{E^{P}}{\varepsilon_{0}}+1\right)^{n-1}
$$

Here, $n$ is the hardening exponent, and $\varepsilon_{0}=\sigma_{Y} / E$ is the yield strain in uniaxial tension.

\section{Results}

In the analyzes to be presented here the basic elastic-plastic material parameters are taken to have the values $\sigma_{Y} / E=0.003, v=0.3$ and $N=0.1$. Two different values of the ratio $R_{0} / X_{0}$ of the initial void radius to void spacing are considered, to study both the void by void mechanism of crack growth and the multiple 
void mechanism. In all cases the value of the initial crack-tip radius is taken to be specified by $r_{0} / X_{0}=0.0667$, while the effect of different values of the characteristic material length scale is studied. The computations are stopped well before the crack would actually grow by coalescence of the first void with the blunting crack-tip, since a realistic description of such failure would require remeshing, as applied in [12].

In the first case analyzed the initial void size is specified by $R_{0} / X_{0}=0.02$ so that the initial void volume fraction in the strip of material of width $X_{0}$ ahead of the crack-tip is $f_{0}=0.00126$. The material model using a single material length parameter is applied, with the characteristic length parameter in (2) specified by $l_{*} / R_{0}=1.0$. It is chosen to stop the computation at $J / \sigma_{Y} X_{0}=1.10$ in order to avoid too much mesh distortion around the growing voids. Fig. 2 shows the initial mesh and the subsequent deformed mesh at $J / \sigma_{Y} X_{0}=1.10$, together with the contours of the plastic strain $\varepsilon^{P}$ at the latter stage. Fig. 3 shows the evolution of the four void volumes normalized by the initial void volumes $V_{0}$, as functions of $J / \sigma_{Y} X_{0}$. It is seen both in Figs. $2 \mathrm{~b}$ and $2 \mathrm{c}$ and in in Fig. 3 that the void closest to the blunting crack-tip has grown a lot, while the other three voids have hardly grown at all. It is clear that this case is dominated by the void by void mechanism.

In Fig. 2c the curves for $\varepsilon^{P}=0.001$ indicate the edges of the plastic zone, which has the usual shape for a plane strain mode 1 crack when seen on a larger scale. The much larger strain values shown in Fig. 2c are features associated with the blunting crack-tip and the void growing near the tip. It should be noted that with the special two-field finite elements applied here it was found necessary to use a rather fine mesh in the region near the voids, finer than meshes used in the previous studies for standard plasticity theory. Analyses were carried out with different mesh refinement, and good convergence was found for larger values of $l_{*} / R_{0}$, but the fine mesh was needed for the value $l_{*} / R_{0}=1.0$ considered in Figs. 2 and 3 . The situation is worse for smaller $l_{*} / R_{0}$ and therefore no results are shown here for $l_{*} / R_{0}=0$, but comparison with results for standard local plasticity will be mentioned.

Fig. 4 shows curves like those in Fig. 3 for a case where the characteristic material length is larger $l_{*} / R_{0}=2.0$, but where all other material parameters are identical to those considered in the first case analyzed. As is seen by comparison of the two figures, the larger value of $l_{*} / R_{0}$ has the expected effect that much less void growth has occurred at the same value of $J / \sigma_{Y} X_{0}$. Fig. 5 gives a comparison of five different analyzes, for five different values of $l_{*} / R_{0}$. The numbers 1 to 4 on the horizontal axis indicate void No. 1 to 4 , the points of the curves above each number indicate the normalized value of the void volume at $J / \sigma_{Y} X_{0}=1.10$ and the four points for each computation are connected by straight lines. In Fig. 5 only void No. 1 has had any significant void growth, so that here the void by void crack growth mechanism is dominant in all cases. However, for the largest value of $l_{*} / R_{0}$ very little void growth is seen at all in the range considered.

To consider the case $l_{*} / R_{0}=0$ a computation with the mesh in Fig. 2 a has been carried out for standard local $J_{2}$ flow theory. This computation broke down numerically before reaching the stage $J / \sigma_{Y} X_{0}=1.10$, due to mesh distortion at void No. 1. However, at $J / \sigma_{Y} X_{0}=1.0$ the value of $V_{1} / V_{0}$ had reached 27.4, about twice the value found in Fig. 3, while the other three voids had grown very little. Assuming that the value of $V_{1} / V_{0}$ in Fig. 5 would be about two times that for $l_{*} / R_{0}=1.0$ this agrees with the trend found by comparing the results for the values $2.0,1.5$ and 1.0 of $l_{*} / R_{0}$, where the numerical predictions were 
found to converge. But the value for $l_{*} / R_{0}=0.5$ appears to be too small, as expected due to the very slow convergence for the smaller values of $l_{*} / R_{0}$.

Fig. 6 illustrates an analysis for a larger initial void size specified by $R_{0} / X_{0}=0.0667$, corresponding to the initial void volume fraction $f_{0}=0.0140$ in the strip of material of width $X_{0}$ ahead of the crack-tip. The value of the characteristic length parameter in (2) is here taken to be larger relative to the void spacing $X_{0}$, so that again $l_{*} / R_{0}=1.0$. In this case it is chosen to stop the computation at $J / \sigma_{Y} X_{0}=0.75$ to avoid mesh distortion around the growing voids. Fig. 6 shows the initial mesh and the subsequent deformed mesh at $J / \sigma_{Y} X_{0}=0.75$, together with the contours of the plastic strain $\varepsilon^{P}$. In Fig. 7 shows the corresponding evolution of the four normalized void volumes is illustrated. It is seen both in Figs. 6 and 7 that here all the voids grow large, so that here the multiple void mechanism of crack growth is getting activated.

Curves like those in Fig. 7 are presented in Fig. 8 for $l_{*} / R_{0}=1.5$, with all other material parameters identical to those considered in Fig. 7. Also here comparison of the two figures shows that the larger value of $l_{*} / R_{0}$ results in much less void growth at the same value of $J / \sigma_{Y} X_{0}$. Fig. 9 gives a comparison of four different analyzes, for different values of $l_{*} / R_{0}$. As in Fig. 5 voids No. 1 to 4 are indicated on the horizontal axis and the points of the curves above each number indicate the normalized value of the void volume at $J / \sigma_{Y} X_{0}=0.75$. Two of the curves correspond to the end points in Figs. 7 and 8 . It is seen that there is more void growth for $l_{*} / R_{0}=0.5$ than for $l_{*} / R_{0}=1.0$, but otherwise these two curves are nearly parallel, indicating that the multiple void mechanism is active. For $l_{*} / R_{0}=1.5$ (as in Fig. 8) the shape of the curve has changed to something more like the curves in Fig. 5, so in this case the effect of the characteristic material length is to move the behaviour towards the void by void mechanism of crack growth. This is even more so for $l_{*} / R_{0}=2.0$, but at the same time the rate of growth of all the voids is rather strongly suppressed at the stage shown.

A computation for the same mesh using standard $J_{2}$ flow theory gave $V_{1} / V_{0}=20.3$ at $J / \sigma_{Y} X_{0}=0.75$, again about two times the value found for $l_{*} / R_{0}=1.0$ in Fig. 9. Also here the value of $V_{1} / V_{0}$ found for $l_{*} / R_{0}=0.5$ is well below the value found by interpolation between $l_{*} / R_{0}=1.0$ and $l_{*} / R_{0}=0.0$.

A few computations have also been made using the nonlocal model with three material length parameters, according to (1). Fig. 10 gives curves like those in Fig. 5, indicating the normalized values of the void volumes at $J / \sigma_{Y} X_{0}=1.10$. The curve based on the single parameter theory for $l_{*} / R_{0}=2.0$ is repeated from Fig. 5. As is expected based on previous results for void growth [14,16], predictions obtained by using $l_{1} / R_{0}=1.0$ are rather close to those obtained for the double value of $l_{*}$, such that there is slightly less constraint on void growth for $l_{1} / R_{0}=1.0$. In the previous void growth studies the influence of $l_{2}$ and $l_{3}$ was found negligible, but this is not true in the present study where shear deformations in the crack-tip region play a significant role. Thus, the curve for $l_{2} / R_{0}=l_{3} / R_{0}=1.0$ shows noticeably less void growth than that for $l_{2}=l_{3}=0.0$.

. Fig. 11 gives curves like those in Fig. 9, indicating the normalized values of the void volumes at $J / \sigma_{Y} X_{0}=0.75$. As in Fig. 10 a curve based on the single parameter theory, for $l_{*} / R_{0}=1.0$, is compared to curves for half the value of $l_{1} / R_{0}$, and again these curves are rather close to each other. As in Fig. 10 the 
influence of $l_{2}$ and $l_{3}$ is not found negligible, as the curve for $l_{2}=l_{3}=0.0$ shows noticeably more void growth than that for $l_{2} / R_{0}=l_{3} / R_{0}=0.5$.

Results analogous to those in Fig. 6 are illustrated in Fig. 12 with the only difference that here the material length is much larger compared to the initial void radii and the initial crack-tip radius, $l_{*} / R_{0}=10.0$. As in Fig. 6 the computation has been stopped at $J / \sigma_{Y} X_{0}=0.75$, and the deformed mesh in Fig. 12b as well as the corresponding plastic strain contours in Fig. 12c show that the voids have hardly grown at all at this stage. But here the value of $l_{*} / r_{0}$ is large enough so that the rate of increase of the blunting radius is noticeably reduced by the strain gradient dependence of the constitutive model, as seen by comparing Figs. $12 \mathrm{~b}$ and $6 \mathrm{~b}$. Also, the highest plastic strain contour at the crack-tip shown in Fig. $12 \mathrm{c}$ is $\varepsilon^{P}=0.2$, while the highest value in Fig. 6c is $\varepsilon^{P}=1.4$. This gives only an indication of the effect of gradient plasticity on an initially sharp crack-tip, as studied by Komaragiri et al. [25].

\section{Discussion}

The present analyses of void growth in the vicinity of a blunting crack-tip have shown the expected effect of strain gradient dependent plasticity that voids grow more slowly when the characteristic material length is comparable to or larger than the void radius. Thus, for values of $l_{*} / R_{0}$ or $l_{i} / R_{0}$ well above unity the voids hardly grow at all in the ranges considered here. Since experiments have shown that the characteristic material length is of the order of $0.5 \mu \mathrm{m}$ to $5 \mu \mathrm{m}$ [27], it is quite realistic that the void radius could be smaller than the material length. On the other hand, when there is void growth the ratio of the material length and the current void radius is gradually reduced so that after some growth the gradient terms give only little constraint on the void growth rate.

When the voids ahead of the blunting crack-tip are sufficiently small so that standard plasticity theory would predict interaction only with the nearest void, this result is also found in the analyses where effects of plastic strain gradients are incorporated (Fig. 5). For increasing values of $l_{*} / R_{0}$ the rate of growth of the nearest void is reduced, but the general picture remains the same, such that the early part of the growth pattern analyzed here indicates that crack growth will occur by the void by void mechanism. However, when the voids ahead of the crack-tip are initially larger the growth pattern is more affected by the strain gradient plasticity. Here, when the value of $l_{*} / R_{0}$ increases from 1.0 to 1.5 , the growth rates of the four voids considered change to a pattern more like that found for the initially smaller voids (Fig. 9). An important mechanism here would be that the nearest void, forced to open by the near vicinity to the crack-tip, will gradually outgrow the strain gradient constraint on the growth rate as the current radius increases above the value of $l_{*}$, whereas the following voids still have the full constraint.

The effect of strain gradients also reduces the rate of growth of the crack-tip radius during blunting. For the initial geometries considered here this requires much larger values of $l_{*} / R_{0}$, as seen in Fig. 12. For an initially sharp crack-tip Komaragiri et al. [25] have illustrated this dependence by showing how a normalized crack opening displacement at a distance $0.01 R_{p}$ behind the tip increases for increasing value of $R_{p} / l_{*}$, where $R_{p}$ denotes the plastic zone size. 
When comparing the single parameter nonlocal model with the three parameter model (Figs. 10 and 11) a significant difference from previous void growth studies is observed. In cell model studies for a single void it has been found that non-zero values of $l_{2}$ and $l_{3}$ have practically no effect, since there are no or nearly no shear strains. However, for voids growing in crack-tip fields shear strains play an important role, so that not only $l_{1}$, but also $l_{2}$ and $l_{3}$ give a noticeable reduction in the rate of void growth.

A complete study of the interaction of several characteristic lengths involved in the present problem would require many more computations where also the initial crack-tip radius and the void spacings were varied independently, while the other characteristic lengths were kept constant. Here the focus has been on variations of the material lengths in the nonlocal plasticity theory and the initial void radii, while the last two characteristic lengths of the problem have been kept constant.

\section{Conclusion}

The result that voids grow more slowly when the characteristic material length is comparable to or larger than the void radius is also found in the strongly nonuniform strain field at the tip of a crack.

For the strain gradient plasticity model with three lengths it is found that not only $l_{1}$, but also $l_{2}$ and $l_{3}$ reduce the rate of void growth, due to the importance of shear in the crack-tip fields.

The competition between the void by void mechanism of crack growth and the multiple void mechanism is affected by the nonlocal plasticity model in the case of relatively large voids xompared to the void spacing ahead of the crack-tip, where the latter mechanism is active in the absence of strain gradient effects. Here, it has been found that an increasing value of $l_{*} / R_{0}$ can change the behaviour towards the void by void mechanism.

\section{References}

[1] J.R. Rice and M.A. Johnson, in Inelastic behavior of solids, edited by M.F. Kanninen et al., (McGrawHill, New York 1970).

[2] R.M. McMeeking, J. Mech. Phys. Solids 25357 (1977).

[3] N. Aravas and R.M. McMeeking, J. Mech. Phys. Solids 3325 (1985).

[4] C.L. Hom and R.M. McMeeking, (1989) J. Mech. Phys. Solids 37395 (1989).

[5] A.L. Gurson, J. Engng. Mater. Technol. 992 (1977).

[6] A. Needleman and V. Tvergaard, J. Mech. Phys. Solids 35151 (1987). 
[7] G. Rousselier, Nucl. Engng. Des. 10597 (1987).

[8] W.K. Brocks, Klingbeil, D. Kunecke and D.-Z. Sun, in Constraint effects in fracture: theory and applications, edited by M. Kirk and A. Bakker, (ASTM STP 1244, Philadelphia, PA. 1995).

[9] X. Gao, J. Faleskog, C.-F. Shih and R.H. Dodds, Engng. Frac. Mech. 59761 (1998).

[10] V. Tvergaard and A. Needleman, J. Mech. Phys. Solids 40447 (1992).

[11] V. Tvergaard and J.W. Hutchinson, Int. J. Solids Structures 393581 (2002).

[12] V. Tvergaard, Discrete modeling of ductile crack growth by void growth to coalescence. Int. J. Fracture (2008) (to appear).

[13] J.P. Petti and R.H. Dodds Jr., Int.J. Solids Structures 423655 (2005).

[14] N.A. Fleck and J.W. Hutchinson, J. Mech. Phys. Solids 492245 (2001).

[15] Y. Huang, H. Gao, W. Nix and J.W. Hutchinson, J. Mech. Phys. Solids 4899 (2000).

[16] C.F. Niordson and V. Tvergaard, J. Appl. Mech. 73246 (2006).

[17] C.F. Niordson and V. Tvergaard, Modelling Simul. Mater. Sci. Eng., 15:S51-S60, 2007.

[18] C.F. Niordson and P. Redanz, J. Mech. Phys. Solids 522431 (2004).

[19] E.C. Aifantis, ASME J. Eng. Mater. Technol. 106326 (1984).

[20] M. Gurtin and A. Needleman, J. Mech. Phys. Solids 531 (2005).

[21] C.F. Niordson, J. Mech. Phys. Solids 511863 (2003).

[22] C.F. Niordson, On higher-order boundary conditions at elastic-plastic boundary in strain-gradient plasticity, (presented at the same conference).

[23] R. de Borst and H.-B. Mühlhaus, Int. J. Numer. Methods Eng. 35521 (1992).

[24] C. Niordson and V. Tvergaard, Int. J. Solids Structures 422559 (2005).

[25] U. Komaragiri, S.R. Agnew, R.P. Gangloff and M.R. Begley, The Role of Individual Material Length-Scales in Strain Gradient Plasticity Predictions of Crack Tip Behavior, Report, University of Virginia, USA (2007).

[26] L.P. Mikkelsen, (Private Communication).

[27] J.W. Hutchinson, Int. J. Solids Structures 37225 (2000). 


\section{Figure captions:}

Fig. 1. Geometry of the near-tip region in the plane strain small scale yielding model.

Fig. 2. Mesh in the void region for $r_{0} / X_{0}=0.0667$ and $R_{0} / X_{0}=0.02$, i.e. $f_{0}=0.00126$. The material length scale is specified by $l_{*} / R_{0}=1.0$. (a) Initial mesh. (b) Deformed mesh at $J / \sigma_{Y} X_{0}=1.10$. (c) Plastic strain contours at $J / \sigma_{Y} X_{0}=1.10$.

Fig. 3. Evolution of the four void volumes normalized by the initial void volume $V_{0}$, as functions of $J / \sigma_{Y} X_{0}$, for $r_{0} / X_{0}=0.0667$ and $R_{0} / X_{0}=0.02$. The material length scale is specified by $l_{*} / R_{0}=1.0$.

Fig. 4. Evolution of the four void volumes normalized by the initial void volume $V_{0}$, as functions of $J / \sigma_{Y} X_{0}$, for $r_{0} / X_{0}=0.0667$ and $R_{0} / X_{0}=0.02$. The material length scale is specified by $l_{*} / R_{0}=2.0$.

Fig. 5. Normalized values of the void volumes at $J / \sigma_{Y} X_{0}=1.10$, with the four points connected by straight lines, for $r_{0} / X_{0}=0.0667$ and $R_{0} / X_{0}=0.02$.

Fig. 6. Mesh in the void region for $r_{0} / X_{0}=0.0667$ and $R_{0} / X_{0}=0.0667$, i.e. $f_{0}=0.0140$. The material length scale is specified by $l_{*} / R_{0}=1.0$. (a) Initial mesh. (b) Deformed mesh at $J / \sigma_{Y} X_{0}=0.75$. (c) Plastic strain contours at $J / \sigma_{Y} X_{0}=0.75$.

Fig. 7. Evolution of the four void volumes normalized by the initial void volume $V_{0}$, as functions of $J / \sigma_{Y} X_{0}$, for $r_{0} / X_{0}=0.0667$ and $R_{0} / X_{0}=0.0667$. The material length scale is specified by $l_{*} / R_{0}=1.0$.

Fig. 8. Evolution of the four void volumes normalized by the initial void volume $V_{0}$, as functions of $J / \sigma_{Y} X_{0}$, for $r_{0} / X_{0}=0.0667$ and $R_{0} / X_{0}=0.0667$. The material length scale is specified by $l_{*} / R_{0}=1.5$.

Fig. 9. Normalized values of the void volumes at $J / \sigma_{Y} X_{0}=0.75$, with the four points connected by straight lines, for $r_{0} / X_{0}=0.0667$ and $R_{0} / X_{0}=0.0667$.

Fig. 10. Normalized values of the void volumes at $J / \sigma_{Y} X_{0}=1.10$, with the four points connected by straight lines, for $r_{0} / X_{0}=0.0667$ and $R_{0} / X_{0}=0.02$. The general model (1) with three different length parameters is compared with that for one parameter.

Fig. 11. Normalized values of the void volumes at $J / \sigma_{Y} X_{0}=0.75$, with the four points connected by straight lines, for $r_{0} / X_{0}=0.0667$ and $R_{0} / X_{0}=0.0667$. The general model (1) with three different length parameters is compared with that for one parameter. 
Fig. 12. Mesh in the void region for $r_{0} / X_{0}=0.0667$ and $R_{0} / X_{0}=0.0667$, i.e. $f_{0}=0.0140$. The material length scale is specified by $l_{*} / R_{0}=10.0$. (a) Initial mesh. (b) Deformed mesh at $J / \sigma_{Y} X_{0}=0.75$. (c) Plastic strain contours at $J / \sigma_{Y} X_{0}=0.75$. 


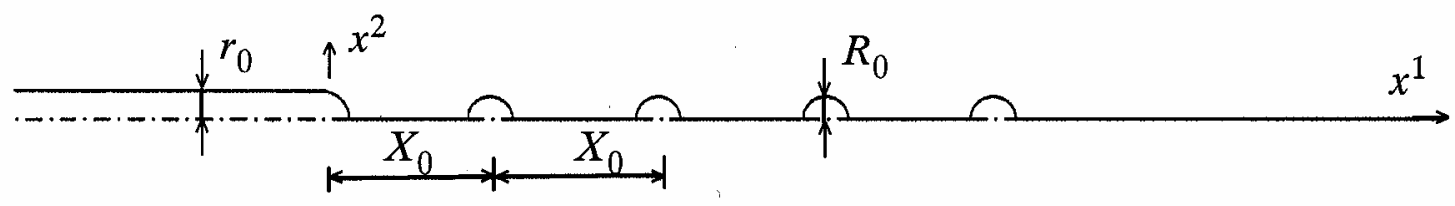

Fig. 1. Geometry of the near-tip region in the plane strain small scale yielding model.

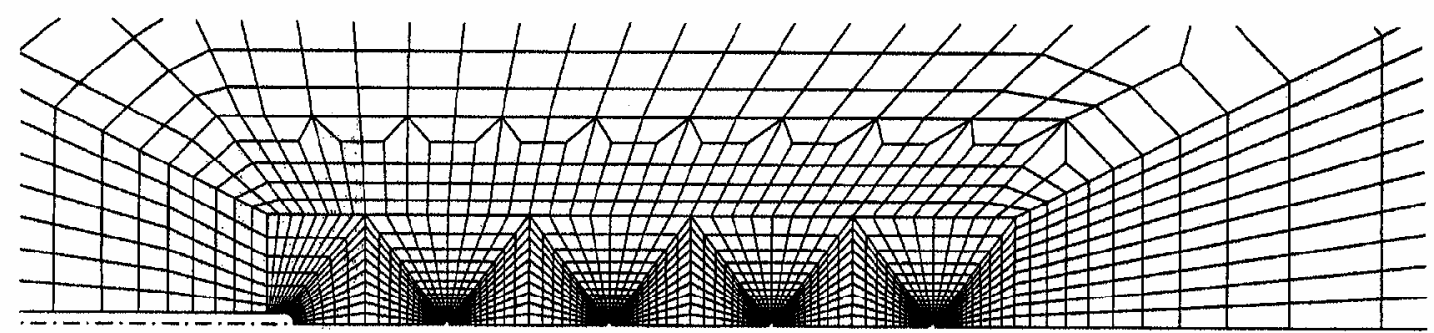

(a)

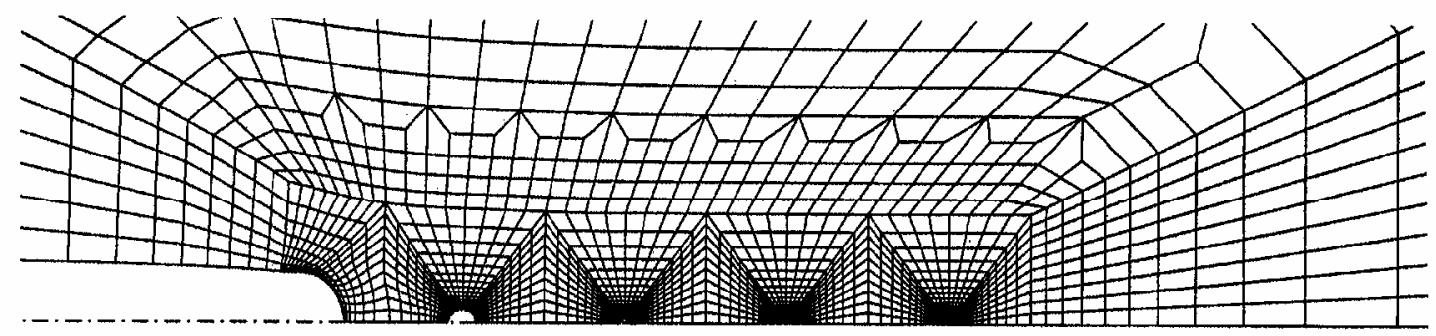

(b)

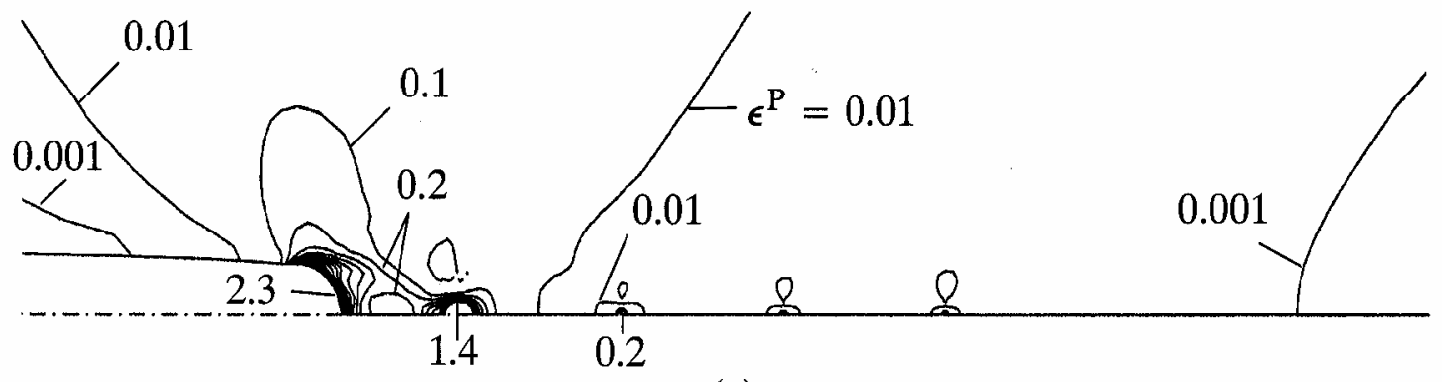

(c)

Fig. 2. Mesh in the void region for $r_{0} / X_{0}=0.0667$ and $R_{0} / X_{0}=0.02$, i.e. $f_{0}=0.00126$. The material length scale is specified by $l_{*} / R_{0}=1.0$. (a) Initial mesh. (b) Deformed mesh at $J / \sigma_{Y} X_{0}=1.10$. (c) Plastic strain contours at $J / \sigma_{Y} X_{0}=1.10$. 


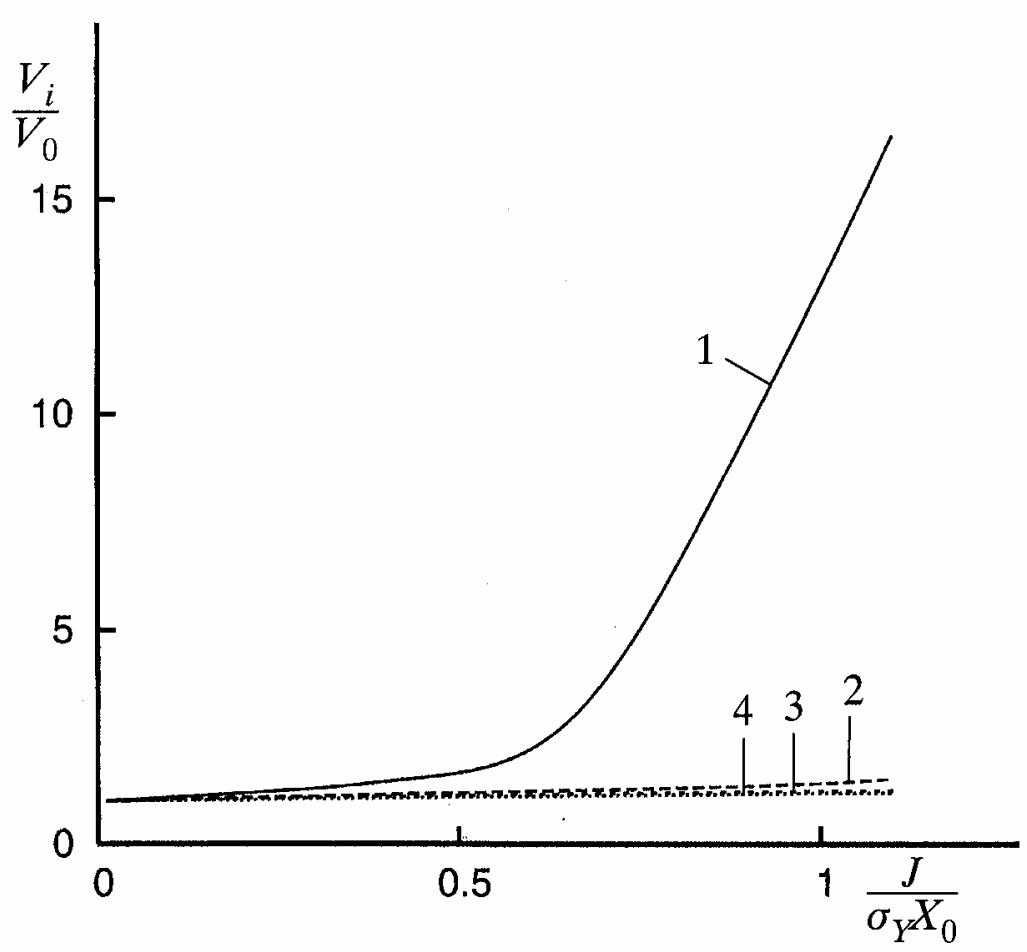

Fig. 3. Evolution of the four void volumes normalized by the initial void volume $V_{0}$, as functions of $J / \sigma_{Y} X_{0}$, for $r_{0} / X_{0}=0.0667$ and $R_{0} / X_{0}=0.02$. The material length scale is specified by $l_{*} / R_{0}=1.0$.

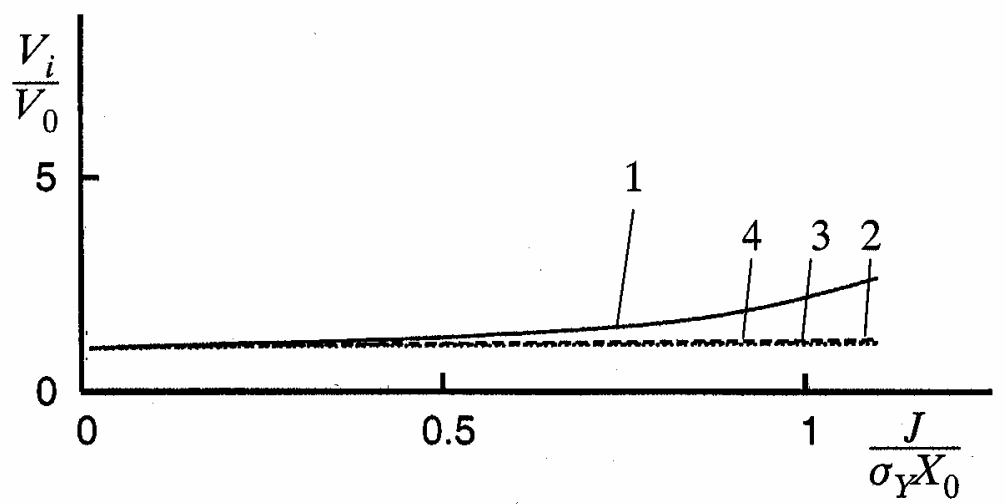

Fig. 4. Evolution of the four void volumes normalized by the initial void volume $V_{0}$, as functions of $J / \sigma_{Y} X_{0}$, for $r_{0} / X_{0}=0.0667$ and $R_{0} / X_{0}=0.02$. The material length scale is specified by $l_{*} / R_{0}=2.0$. 


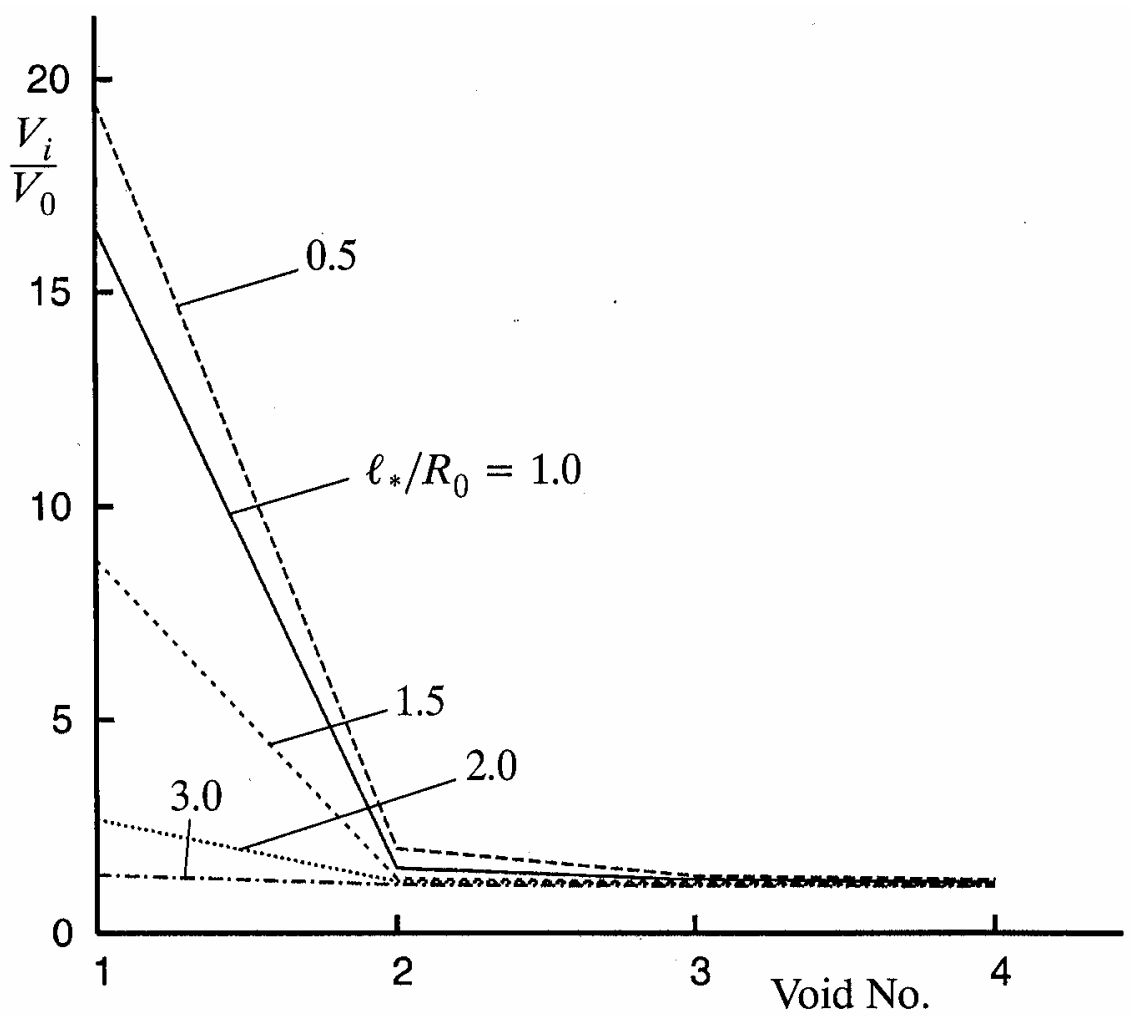

Fig. 5. Normalized values of the void volumes at $J / \sigma_{Y} X_{0}=1.10$, with the four points connected by straight lines, for $r_{0} / X_{0}=0.0667$ and $R_{0} / X_{0}=0.02$. 


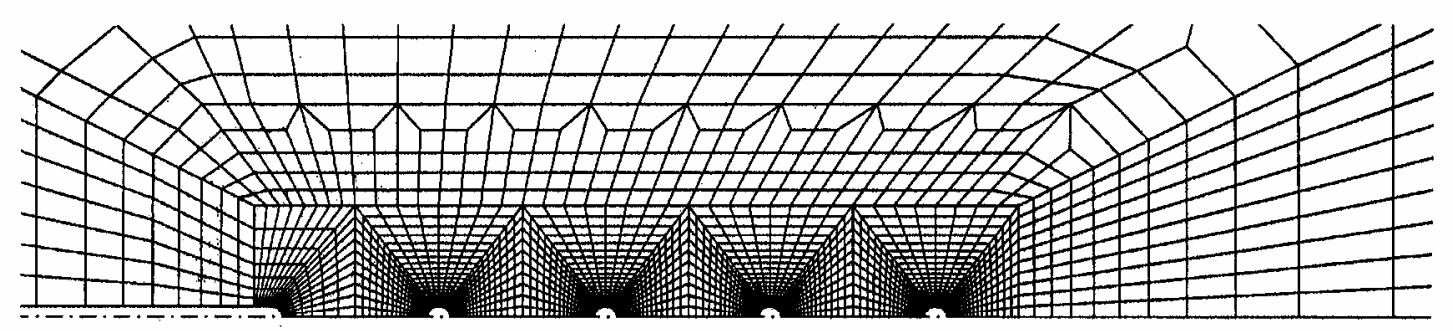

(a)

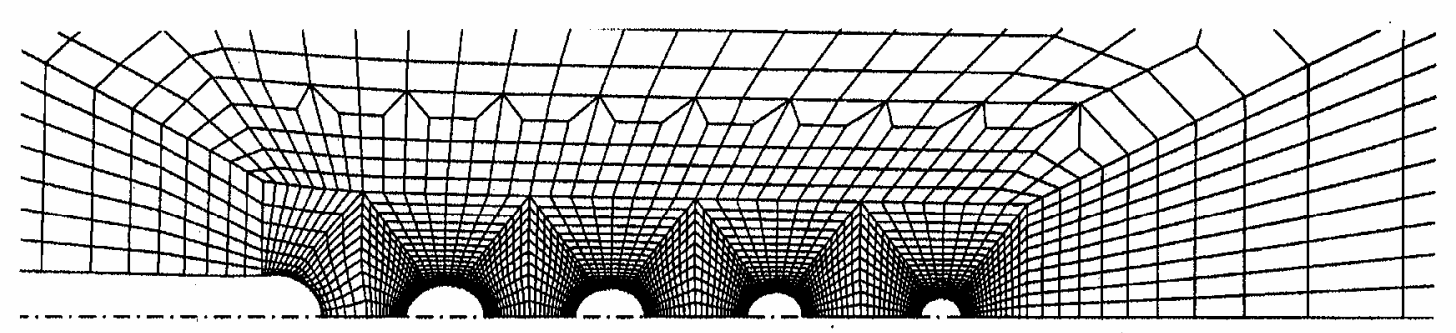

(b)

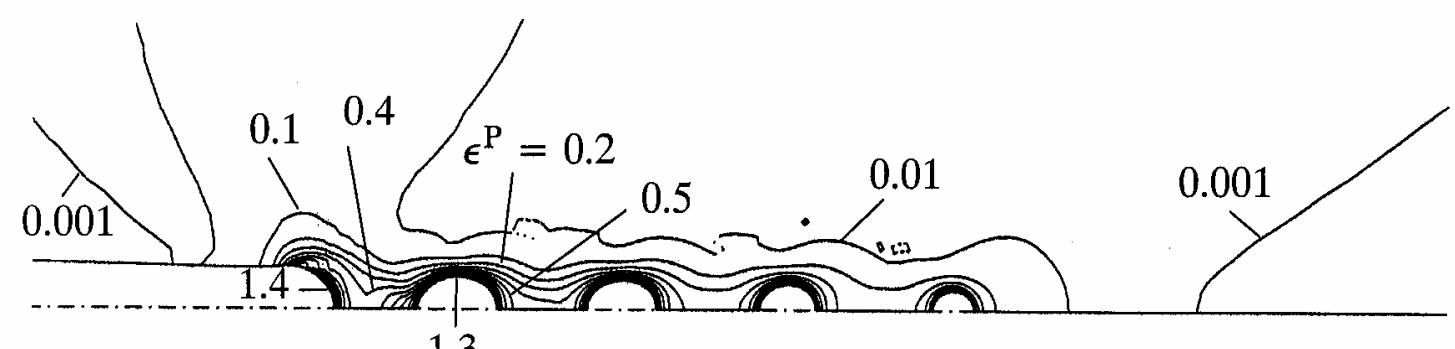

(c)

Fig. 6. Mesh in the void region for $r_{0} / X_{0}=0.0667$ and $R_{0} / X_{0}=0.0667$, i.e. $f_{0}=0.0140$. The material length scale is specified by $l_{*} / R_{0}=1.0$. (a) Initial mesh. (b) Deformed mesh at $J / \sigma_{Y} X_{0}=0.75$. (c) Plastic strain contours at $J / \sigma_{Y} X_{0}=0.75$. 


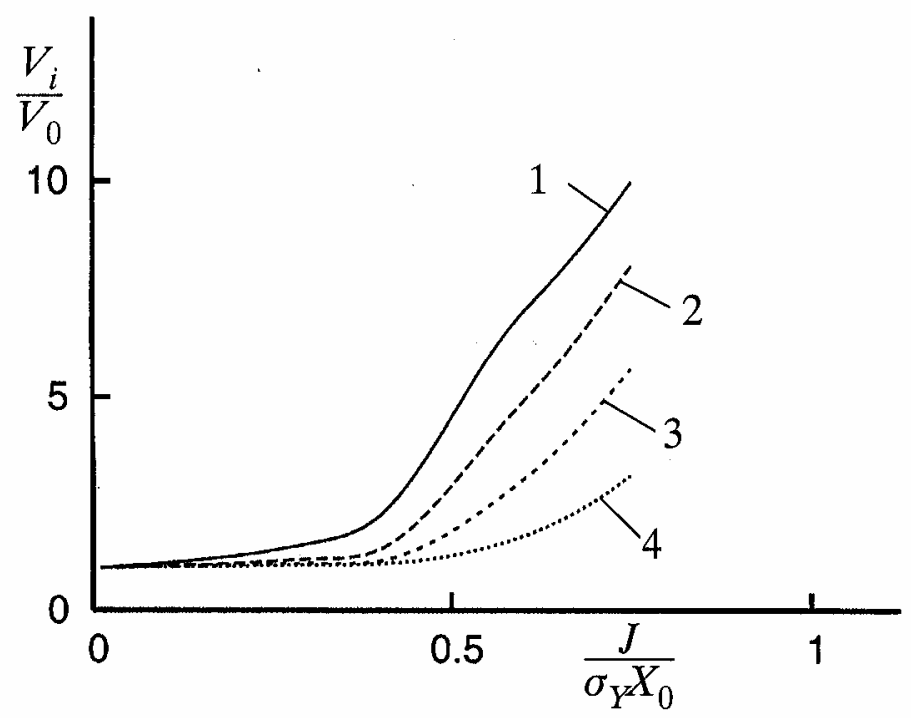

Fig. 7. Evolution of the four void volumes normalized by the initial void volume $V_{0}$, as functions of $J / \sigma_{Y} X_{0}$, for $r_{0} / X_{0}=0.0667$ and $R_{0} / X_{0}=0.0667$. The material length scale is specified by $l_{*} / R_{0}=1.0$.

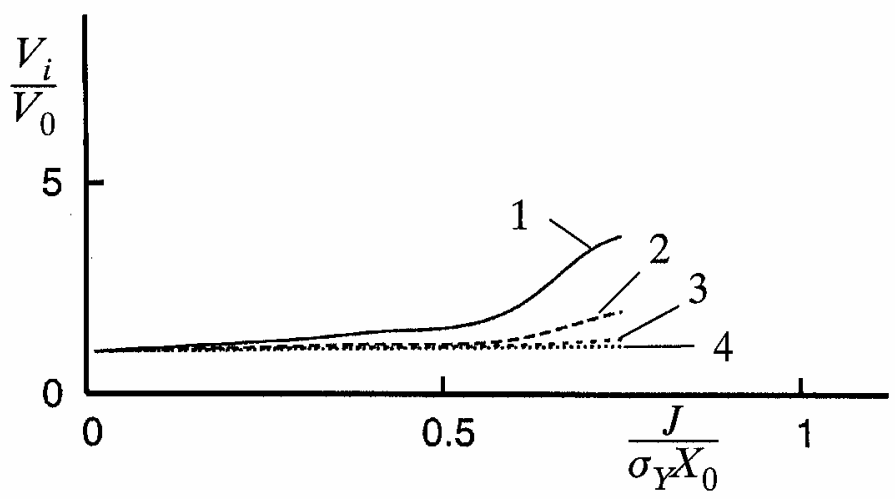

Fig. 8. Evolution of the four void volumes normalized by the initial void volume $V_{0}$, as functions of $J / \sigma_{Y} X_{0}$, for $r_{0} / X_{0}=0.0667$ and $R_{0} / X_{0}=0.0667$. The material length scale is specified by $l_{*} / R_{0}=1.5$. 


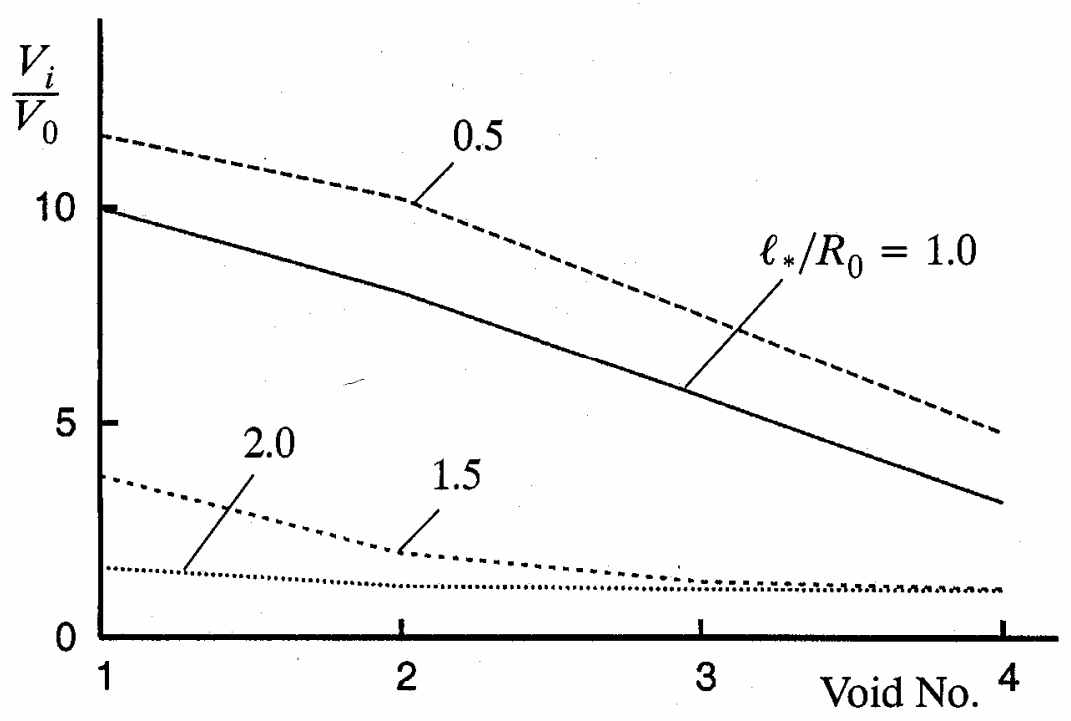

Fig. 9. Normalized values of the void volumes at $J / \sigma_{Y} X_{0}=0.75$, with the four points connected by straight lines, for $r_{0} / X_{0}=0.0667$ and $R_{0} / X_{0}=0.0667$.

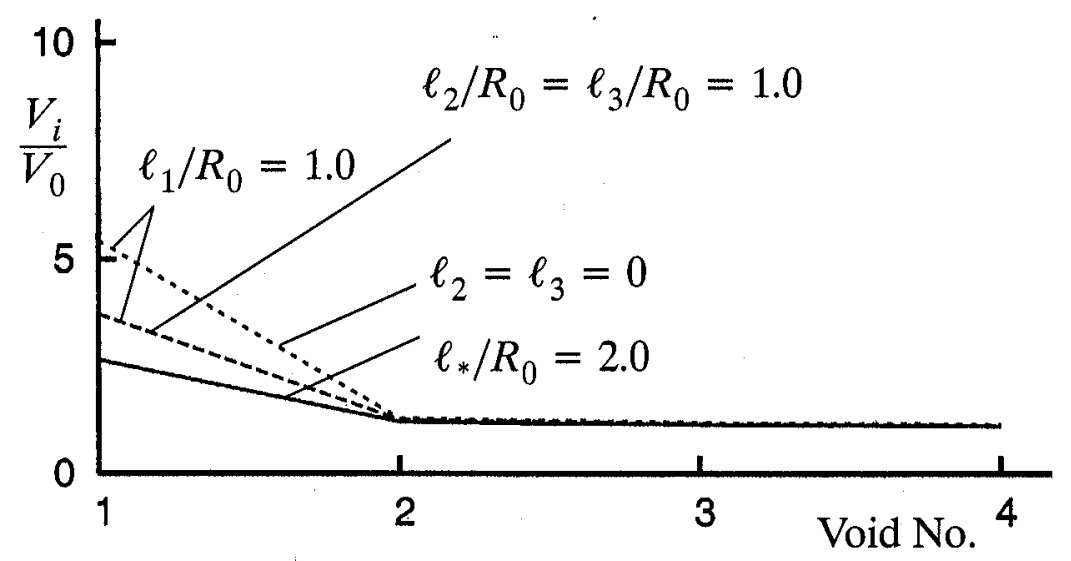

Fig. 10. Normalized values of the void volumes at $J / \sigma_{Y} X_{0}=1.10$, with the four points connected by straight lines, for $r_{0} / X_{0}=0.0667$ and $R_{0} / X_{0}=0.02$. The general model (1) with three different length parameters is compared with that for one parameter. 


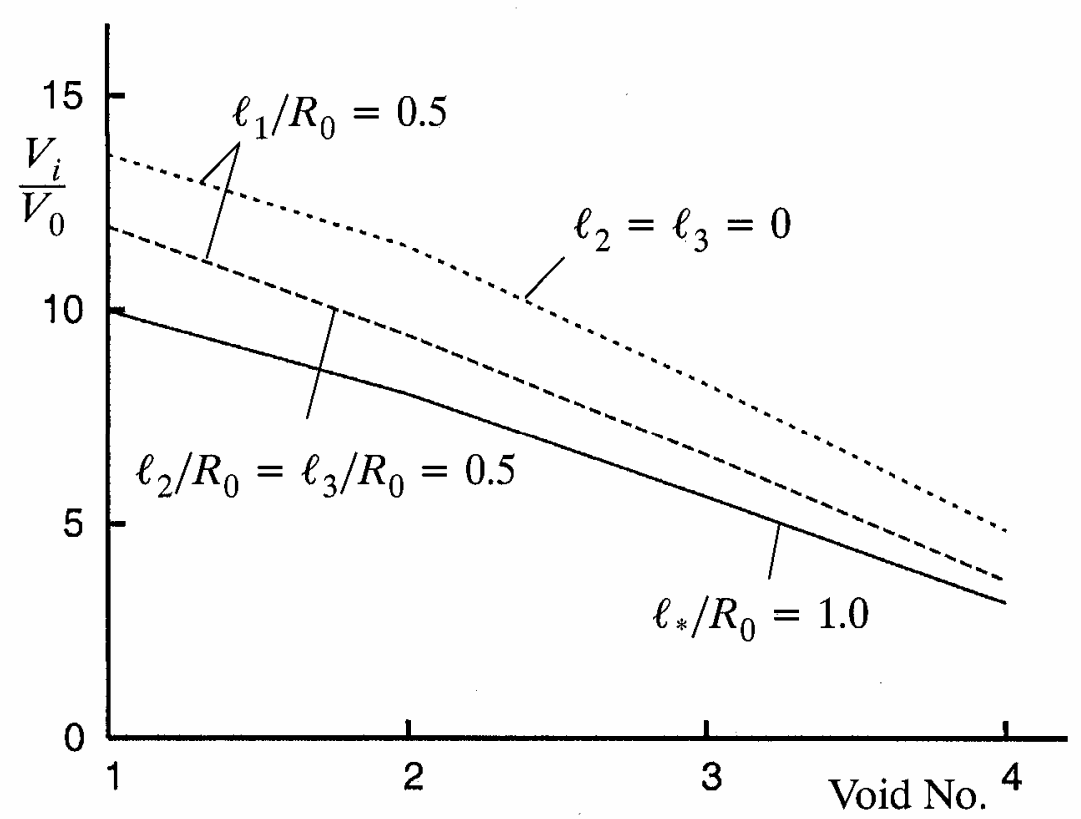

Fig. 11. Normalized values of the void volumes at $J / \sigma_{Y} X_{0}=0.75$, with the four points connected by straight lines, for $r_{0} / X_{0}=0.0667$ and $R_{0} / X_{0}=0.0667$. The general model (1) with three different length parameters is compared with that for one parameter. 


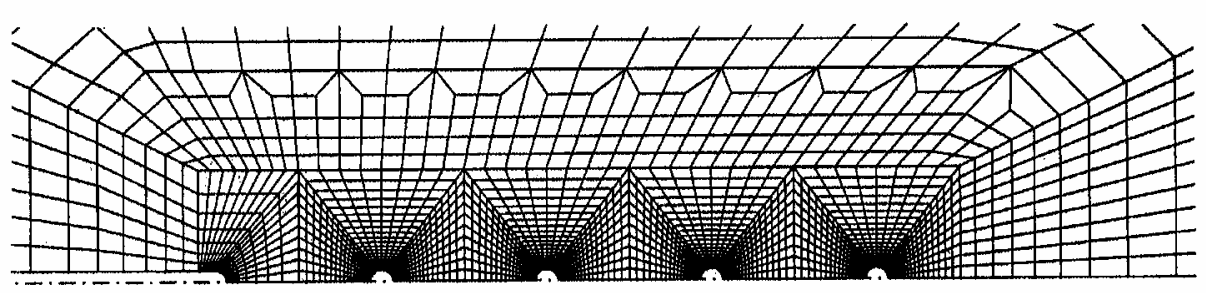

(a)

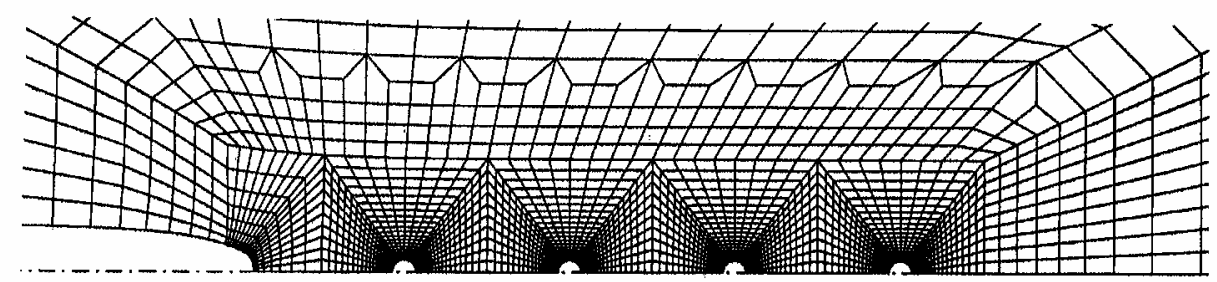

(b)

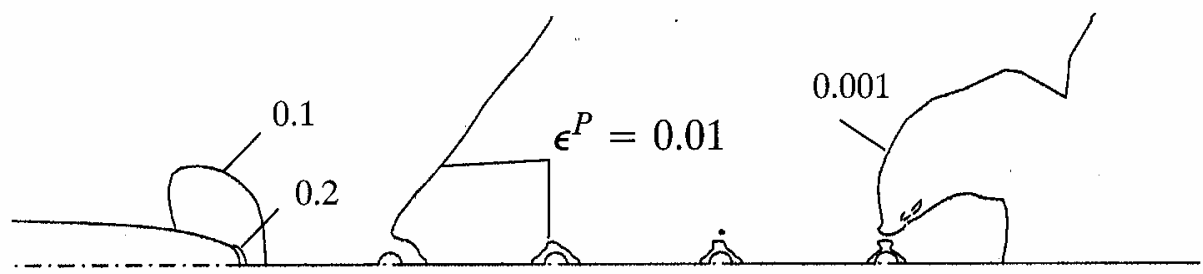

(c)

Fig. 12. Mesh in the void region for $r_{0} / X_{0}=0.0667$ and $R_{0} / X_{0}=0.0667$, i.e. $f_{0}=0.0140$. The material length scale is specified by $l_{*} / R_{0}=10.0$. (a) Initial mesh. (b) Deformed mesh at $J / \sigma_{Y} X_{0}=0.75$. (c) Plastic strain contours at $J / \sigma_{Y} X_{0}=0.75$. 